\title{
Beating cross infection
}

Henry Schein will be exhibiting and demonstrating its range of products on stand H03 this year.

There will be new initiatives in restoratives, cross infection, including new washer decontaminators, and endodontic products. Other services include a practice magazine service, Future Perfect, a practical business learning programme and a BA handpiece repair service.

The range of dental equipment will include dental chairs such as Fedesa chairs and Murray stools, cabinetry to suit all budgets and a wide range of $\mathrm{x}$ ray and imaging equipment. Reader response number 62

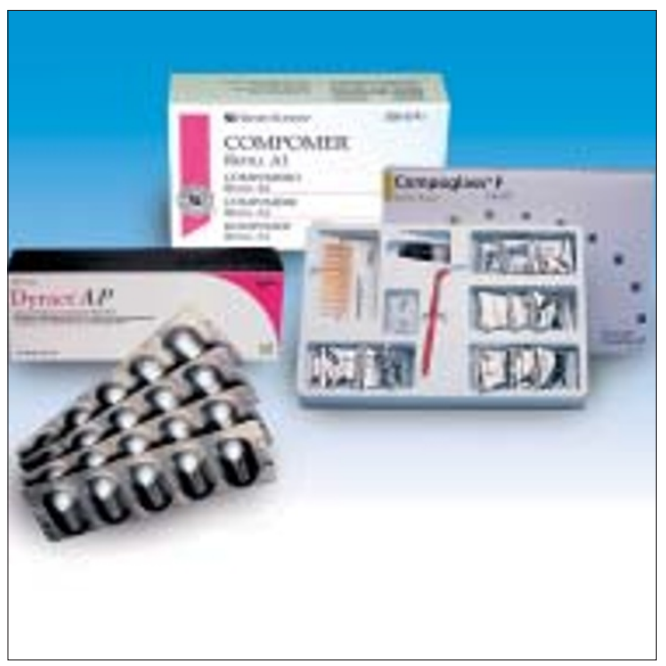

\section{Reducing sensitivity}

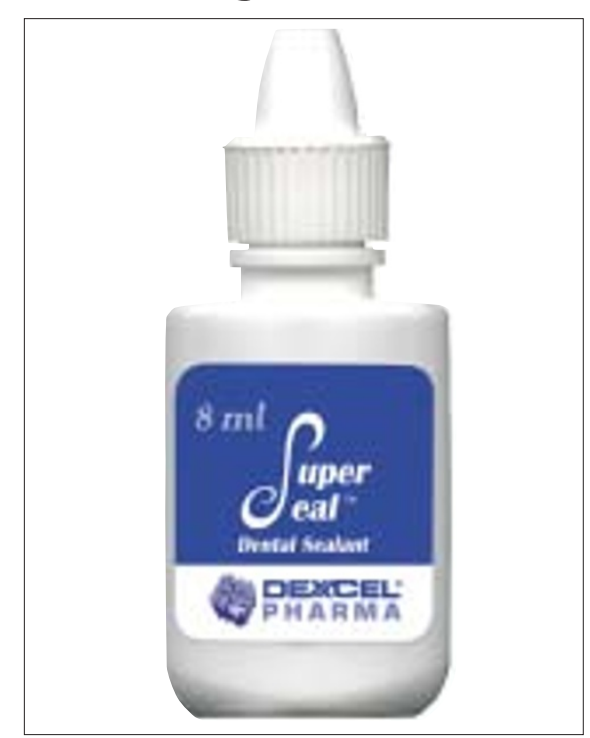

\section{In the mix}

Minerva Dental Limited will be demonstrating their latest innovations on stand F04.

This will include the new developments from DMG which are supplied in their unique MixStar, AutoMix or SmartMix presentations.

There will also be demonstrations of Stick Tech's fibre reinforcement products, including everStick Perio and the opportunity to evaluate Frasaco Models, Omnia Barrier Products, Hannenkratt post systems, Polaroid Macro 5 and Macro 3 instant medical cameras, and the complete range of Tokuyama bonding, chairside relining and resin luting materials.

Also on display will be the range of 'value for money' Codent products. Minerva will also be showcasing the latest Sirona C4+ and recently launched C8+ Dentist and ProFeel Hygienist Treatment Centres, panoramic and intra-oral $\mathrm{x}$-ray
Dexcel Dental will be exhibiting its PerioChip and SuperSeal product range at the Dental Exhibition on stand J14.

SuperSeal is a new water-based, potassium oxalate desensitiser that offers fast, effective desensitisation for years from a single application. It is non-toxic and will not discolour teeth, is easy to apply and biologically compatible with soft tissues.

The product uses calcium within the dentine tubule to form insoluble calcium oxalate crystals that completely block the tubules. It can reduce dentine hypersensitivity in a number of treatments and post treatment situations, including restorations, temporary crowns, under composite fillings, and following tooth whitening treatment.

Reader response number 63

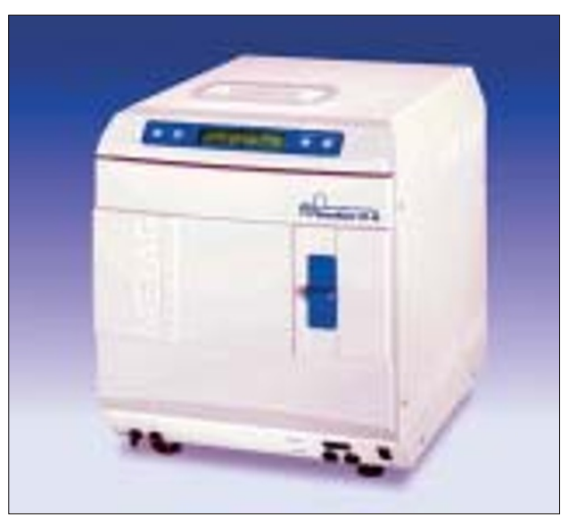

units with digital variants and the new Sironiti Endo Handpiece. Other highlights will include Instrumentarium Imaging's panoramic and intra-oral x-ray units with digital variants, Melag autoclaves, plus cabinetry and the Orbit ambidextrous concept from Minerva.

Reader response number 64 